Some of these conclusions confirm those previously reported by other workers; others are new. They will be published in detail elsewhere.

It is not our object in this communication to relate the experimental results to any of the existing divergent theories. The final test of any theory, however, must be based on detailed qualitative and quantitative experimental data.

$$
\begin{array}{cl}
\text { Davy Faraday Laboratory, } & \text { K. Lonsdale. } \\
\text { Albemarle Street, } & \text { I. E. KNAGGs. } \\
\text { London, W.1. } & \text { H. SMITH. }
\end{array}
$$

1 Nature, 146, 130 (1940)

'Lonsdale, K., and Smith, H., Phil. Mag. (7), 28, 614 (1939).

\section{Dimorphism of Diphenyl Octatetraene Crystals}

The crystal structures of the diphenyl polyenes, having the general formula $\left\langle>-(\mathrm{CH}=\mathrm{CH})_{n}\right\rangle$, have been studied by Hengstenberg and $\mathrm{Kuhn}^{1}$ by $\mathrm{X}$-ray methods. They find that while the lower members of the series, with $n=1$ to 4 , crystallize in the monoclinic system, the higher members studied by them, for which $n=5$ to 7 , crystallize in the orthorhombic system.

Recently, in the course of some electrical and optical measurements on this important series of compounds possessing conjugate double bonds, we had occasion to study the crystal structure of one of them, namely, diphenyl octatetraene, corresponding to $n=4$. On allowing this substance to crystallize from its solution in acetic ether, we found that in addition to some flaky crystals which were monoclinic and had the axial dimensions described by Hengstenberg and Kuhn, there also appeared in the same crop stout well-developed crystals which were ortho-

\begin{tabular}{|c|c|c|c|}
\hline \multirow{2}{*}{$n$} & $\underbrace{a}$ & $b$ & $c$ \\
\hline & \multicolumn{3}{|c|}{ in $A$. } \\
\hline $\begin{array}{l}5 \\
6 \\
7\end{array}$ & $\begin{array}{l}10 \cdot 25 \\
10 \cdot 20 \\
10 \cdot 2\end{array}$ & $\begin{array}{l}7 \cdot 66 \\
7 \cdot 60 \\
7 \cdot 57\end{array}$ & $\begin{array}{l}21 \cdot 2 \\
23 \cdot 58 \\
25 \cdot 95\end{array}$ \\
\hline
\end{tabular}
rhombic. The chemical identity of the two types of crystals is verified by the observation that both types appear in the crops obtained by the evaporation of the solutions in acetic ether of either of them separately.

The orthorhombic crystal belongs to the bipyramidal class, and its unit cell has the dimensions $a=9 \cdot 95, \quad b=7 \cdot 55, \quad \mathrm{c}=19 \cdot 75 \mathrm{~A} .$, and contains 4 molecules of $\left\langle-(\mathrm{CH}=\mathrm{CH})_{4}\langle\rangle\right.$ These dimensions fit well with the dimensions of the unit cells of the higher members of the series-all of which contain 4 molecules-obtained by Hengstenberg and Kuhn, and reproduced in the accompanying table.

K. S. KrishnaN.

S. L. Chorghade.

T. S. ANANTHAPADMANABHaN.

Indian Association for the

Cultivation of Science,

Calcutta.

June 15.

\section{Study of Amorphous Films by Electron Diffraction}

SINCE it is necessary to maintain the electron camera used in electron diffraction studies in a state of high vacuum, the investigation of solutions is in general very difficult. However, the desiccation of hygroscopic substances like zinc chloride does not take place very rapidly even in high vacua of the order of $10^{-6} \sim 10^{-8} \mathrm{~mm}$. mercury; as a matter of fact, the thin films of such substances give diffraction haloes as well as diffraction rings (see photographs). The purpose of this study is the determination of the inner structure of amorphous films of very concentrated solutions through the diffraction patterns obtained.

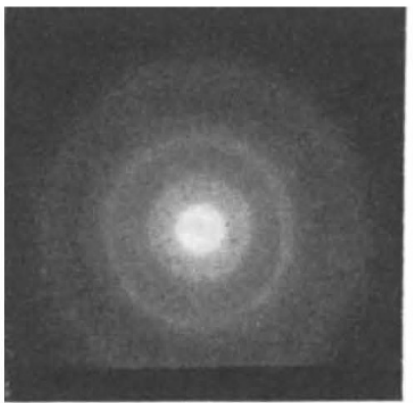

(a)

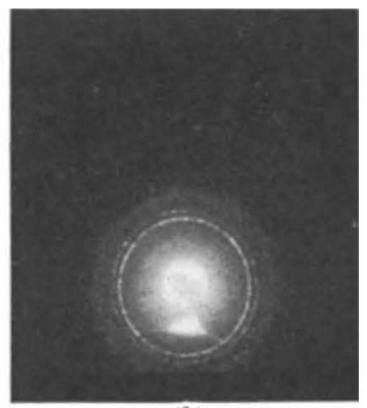

$(b)$
Electron Diffraction patterns from $\mathrm{ZNCL}_{2}$ -

$\mathrm{H}_{2} \mathrm{O}$ FILMS. IN (b) THE RINGS DUE TO $\mathrm{ZNCL}_{2}$ CRYSTALS ARE BEGINNING TO APPEAR.

A platinum wire-gauze of about $0.5 \mathrm{~mm} .{ }^{2}$ dimensions was previously washed with dichromic acid. After immersing the gauze in a saturated aqueous solution of zinc chloride for several hours, a thin film was successfully obtained adhering to every part of the gauze. The films thus obtained were so hygroscopic and had so remarkable a surface effect that they were not easily broken and served satisfactorily for the purpose in the electron diffraction study. Both diffraction patterns shown in the accompanying photographs were obtained by transmission of an electron beam through the $\mathrm{ZnCl}_{2}-\mathrm{H}_{2} \mathrm{O}$ film ; whereas in $a$ haloes are prominent, in $b$ rings are conspicuous. By referring to $\mathrm{X}$-ray data it was clearly verified that these rings were from $\mathrm{ZnCl}_{2}$ crystals (hexagonal, $\mathrm{CdCl}_{2}$ type); therefore, it is clear that the solution under examination is saturated, and its "base-body" is $\mathrm{ZnCl}_{2}$.

If the lattice spacings determined by measuring the radii of haloes remain constant even when the wave-length of the electron beam is altered, it may safely be assumed that the halo pattern obtained is due to the diffraction grating characteristic of the thin film consisting of a saturated solution. The results of the experiment were positive in the several wave-lengths used, namely, 0.0756, 0.0807 and $0.0858 \mathrm{~A}$.; the lattice spacings were respectively $4 \cdot 5, z \cdot 4$ and $1 \cdot 45 \mathrm{~A}$. The haloes are not only sufficiently diffuse to be distinguishable from the sharp rings given by $\mathrm{ZnCl}_{2}$ crystals, but also they appear in the pattern in positions where $\mathrm{ZnCl}_{2}$ crystals cannot produce diffraction rings.

I am now endeavouring to build up a model of the grating characteristic of this film. This work has been conducted under the direction of Dr. Ichirô Iitaka.

Institute of Physical Shigeto YamaguChi. Tokyo. 